\title{
Biominerals for biomacromolecule stabilization and delivery
}

William Murphy

University of Wisconsin, United States

Control over delivery of biomacromolecules (e.g. proteins, DNA, mRNA) is a common theme in natural tissue formation, and also a common theme in emerging biomedical therapies. However, biomacromolecules are often highly unstable, and can rapidly lose their activity due to denaturation, degradation, aggregation, or cell internalization. Indeed, many important proteins in biological processes have half-lives on the order of minutes to hours. In contrast, biomacromolecules embedded in mineralized fossils can remain intact and stable for centuries. We have developed a series of biomineral-based materials that mimic the unique ability of natural fossils to stabilize biomacromolecules. The materials were also designed to dissolve and release their contents over controllable timeframes. In particular, we have synthesized a series of biomineral coatings to deliver genes, growth factors, and stem cells from medical devices. Fundamental mechanisms of affinity binding were used incorporate biomacromolecules into biominerals, and achieved uniquely high stability and biological activity. Stabilization occurred even in extreme environments, such as in the presence of organic solvents, lyophilization, or protease activity. In addition, biomineral coatings were formed on a variety of devices, ranging from 3-D printed scaffolds to injectable microparticles. Coatings were independently optimized for intended biologic delivery without influencing bulk properties of the underlying device. This "modular" approach resulted in devices with optimized properties from the macroscopic scale to the molecular scale. Our recent studies also demonstrated that array-based strategies could select coating chemistries for specific biological or biomedical goals. Examples include coatings that optimize long-term protein stabilization, autologous cell capture, stem cell manufacturing, stem cell differentiation, and gene delivery. Emerging studies focus on efficient local delivery of mRNA therapies, which may have significant impact on vaccine delivery, cancer treatment, and tissue regeneration.

\section{References}

1. A.S. Khalil, X. Yu, P. Dang, E. Alsberg, W.L. Murphy. A microparticle approach for non-viral gene delivery within 3D human mesenchymal stromal cell aggregates. Acta Biomaterialia, 2019; 95: 408-417. 2. A.E. Clements, W.L. Murphy. Injectable biomaterials for delivery of interleukin-1 receptor antagonist: toward improving its therapeutic effect, Acta Biomaterialia, 2019; 93: 123-134.

3. G. Fontana, H.L. Martin, J.S. Lee, K. Schill, P. Hematti, W.L. Murphy. Mineral-coated microparticles enhance mRNA-based transfection of human bone marrow cells. Molecular Therapy - Nucleic Acids, 2019; $18: 455-464$.

4. A.S. Khalil, A.W. Xie, H. Johnson, W.L. Murphy. Sustained release and protein stabilization reduce the growth factor dosage required for human pluripotent stem cell expansion. Biomaterials, 2020; 248: 120007.

5. A.S. Khalil, X.Yu, J.M. Umhoefer, G.M. Diarrad, T.A. Hacker, W.L. Murphy. Single dose mRNA therapy via biomaterial-mediated sequestration of overexpressed proteins. Science Advances, 2020; 6: eaba2422. 